\title{
Children's Collaboration Styles in a Newtonian MicroWorld
}

\author{
Andy Cockburn \\ Department of Computer Science \\ University of Canterbury, Christchurch, N.Z. \\ Tel: +64-3-364-2774 \\ E-mail: andy@ cosc.canterbury.ac.nz
}

\author{
Saul Greenberg \\ Department of Computer Science \\ University of Calgary, Calgary, Canada \\ Tel: +1-403-220-6087 \\ E-mail: saul@cpsc.ucalgary.ca
}

\begin{abstract}
TurboTurtle is a animated multi-user microworld that children use to explore concepts in Newtonian physics. It is a groupware system where students, each on their own computer, can simultaneous control the microworld and gesture in a shared view. Observations of pairs of young children using TurboTurtle highlight extremes in collaboration styles, from conflict to smooth interaction.
\end{abstract}

\section{Keywords}

Computer supported collaborative learning, microworlds.

\section{INTRODUCTION}

Microworlds [3] are computer simulations of restricted environments that promote exploratory learning by children. TurboTurtle (Figure 1) is a microworld that simulates a Newtonian universe [1]. Students explore physical concepts by adjusting properties such as gravity, friction, force, and velocity. They immediately see the effects of these changes on the behavior of a turtle (a ball) that moves through the world. What makes TurboTurtle intriguing is that it is group-aware. Small co-located or distributed groups can talk about the simulation while they are manipulating it. Each student has their own computer screen and input devices. They share the same view of the simulation, have telepointers to facilitate gesturing, and can simultaneously manipulate any aspect of the microworld [1].

We wanted to see how children managed, or mismanaged, their collaboration in this environment that not only allowed parallel activity, but that made no attempt to structure turntaking or mediate conflicting actions.

\section{METHOD}

Twelve children, aged ten or eleven, used the system in mixed sex pairs for 30 minutes. They were observed through think-aloud and constructive interaction techniques. Children were seated approximately two meters apart with a
Cite as:

Cockburn, A. and Greenberg, S.(1996) Children's Collaboration Styles in a Newtonian MicroWorld. ACM SIGCHI'96 Conference on Human Factors in Computing System, Companion Proceedings, p181-182, ACM Press. clear view of each other. They were assigned tasks that familiarized them with TurboTurtle as a collaborative tool, and that progressively introduced the Newtonian concepts of friction, gravity, mass and force.

\section{OBSERVATIONS AND RESULTS}

The children had fun. None left their machine to work directly with their partner on a single machine. Eye contact was rare, but during breakdowns it was common for one child to quickly glance at their partner, without reciprocation. These observations indicate the overall success of TurboTurtle as a shared microworld.

Collaboration styles varied greatly. The summary in Table 1 shows that different pairs talked to each other in quite different ways, and that they manipulated the microworld using various collaboration styles. These are described next.

\section{Collaboration styles}

Parallel activity. Pairs two and five continually discussed their actions and managed their collaborations simultaneously and successfully. As part of this, they were vocal about the undesired actions of their partners. For instance, the boy in pair 2 closed off a rocket control without prior warning. The girl turned to the boy and scolded him with "You aborted the mission!"

Sequential activity. Pair three negotiated control to the near exclusion of simultaneous activity. For example, when asked to set the rocket controls, the girl said "You set the heading, then I'll set the fuel and force. Then you can launch the rocket." The sequence was carried out in that order with no overlapping of actions, and with an explicit "OK" once each stage was completed.

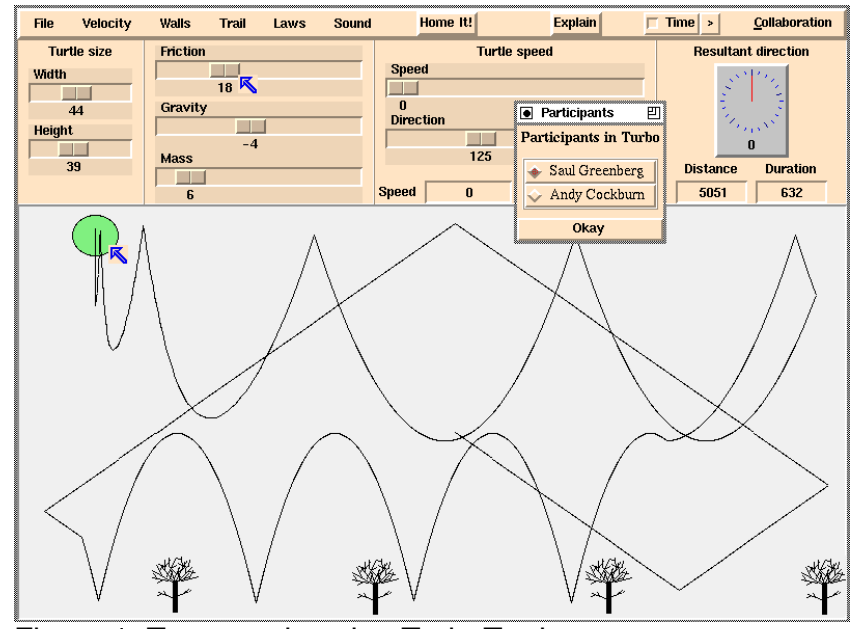

Figure 1. Two people using TurboTurtle. 
Sex Individual style

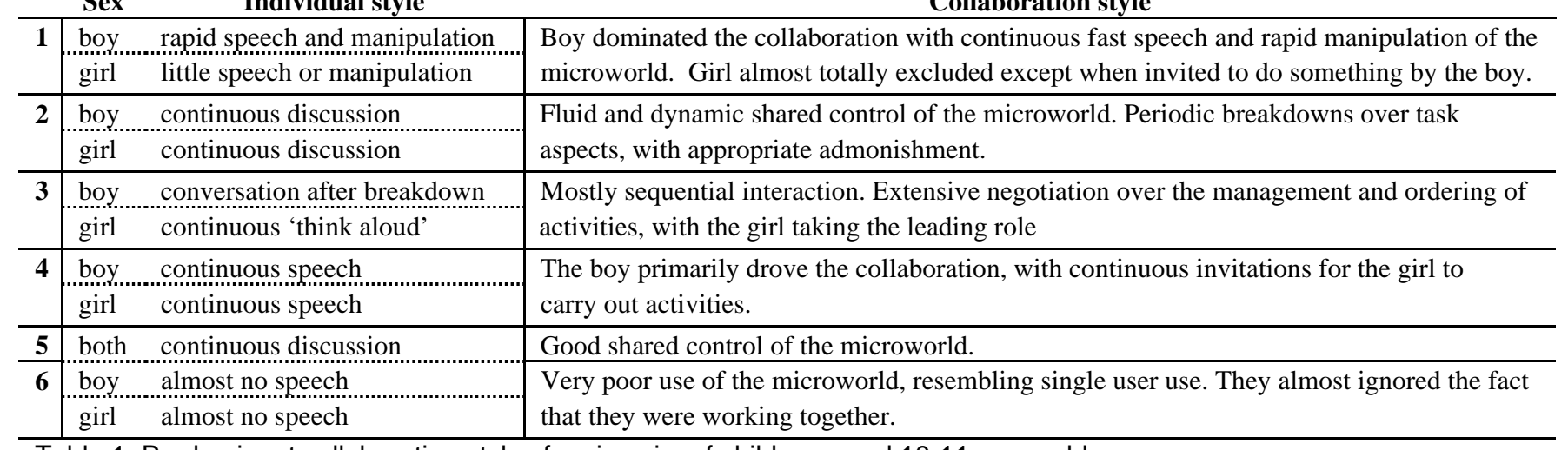

Table 1: Predominant collaboration styles for six pairs of children, aged 10-11 years old.

Independent activity. Pair six almost ignored the fact that they were in a collaborative microworld. They were mostly silent despite being encouraged to communicate. They struggled against the actions of each other, even though the telepointers revealed the cause of their difficulty. They said it would be much easier to use the microworld on their own.

Domination. Breakdowns also occurred when one person dominated the interaction. In pair one, the boy changed the simulation properties so rapidly that the girl could not keep pace. The girl initially took her hands away from the mouse, clearly attempting to follow the frenetic activity of her partner. Shortly afterwards she shouted "Leave it!" While the boy briefly capitulated, he continued to dominate the session, grabbing the controls whenever the girl hesitated.

\section{Breakdowns}

Breakdowns happened even in successful collaborations. Yet many were positive contributions to the overall interaction, with the breakdown becoming a focal point for children negotiating their next manipulation of the microworld. Two factors mitigated breakdown: conversation and mutual awareness.

Conversation. Successful breakdowns were distinguished from unsuccessful ones by the extent of discussion that accompanied the conflict. For instance, pair two argued over the desired mass of the turtle, set by a slider. Their short conflict was accompanied by comments such as "Make it 20!", "No! Make it 30!". Note that the conflict stems from the task, rather than the interface. In contrast, pair six encountered the same problem of simultaneous access to a slider, but it was not clear to them whether the values that they were trying to set were the same or different, the confusion being caused by their total silence.

Mutual awareness. While conflicts over the simultaneous access of sliders were frequent, children were aware of the problem because they saw the two telepointers on the slider as well as the bouncing slider position as both tried to move it. They can then repair the conflict through their natural social skills, much as they do in the real world. This did not always happen. In some cases the children were tenacious in their desire to be last one in control, even though they were well aware of the cause of the problem. This problem arose because of their own immaturity at negotiating control.

Still, the importance of mutual awareness to resolve conflict was emphasized by two bugs. First, in one part of TurboTurtle telepointers are not visible. Frustrated comments such as "Hey, how did that happen," and "What are you doing?" were frequent. Next, some parts of TurboTurtle did not show exactly the same view e.g., pulldown menu actions are not shared, and two pairs said they wanted to be able to see their partner's menu selections. These small breakdowns indicate the importance of keeping aware of another's activities in the microworld.

\section{DISCUSSION AND SUMMARY}

Collaborations worked when children negotiated their interactions, and used mutual awareness and breakdowns to further their discussions. They were less effective when children dominated one another, ignored each other, or fought for control of microworld objects. Yet unlike other microworlds, these problems did not arise because children shared a single input device and display. We agree with Cole [2], who interprets children's control of their collaborations in the microworld to be a social process developed through their own group dynamics. The implication is that groupware microworlds should give children both the freedom to explore the simulation at their own pace and personal style, while adding appropriate structure to minimize the risk of detrimental breakdown that occurs because children are immature collaborators.

\section{REFERENCES}

1. Cockburn, A. and Greenberg, S. (1995) TurboTurtle: A collaborative microworld for exploring Newtonian physics. Proc Computer Supported Collaborative Learning, pp. 32-39, Oct 17-20, LEA Press.

2. Cole, K. (1995) Equality issues in computer based collaborations: Looking beyond surface indicators. Proc Computer Supported Collaborative Learning, pp. 64-74, Oct 17-20, LEA Press.

3. Papert, S. (1993) The children's machine: Rethinking school in the age of the computer. Basic Books. 